\title{
AN ERGODIC LEMMA
}

\section{A. AKCOGLU 1}

Recently, A. Brunel proved an ergodic lemma [1] and applied it to give another proof of the following theorem [2].

Theorem 1. Let $(X, \mathfrak{F}, \mu)$ be a $\sigma$-finite measure space and let $T$ be a positive linear operator in $L_{1}(X, \mathfrak{F}, \mu)$ with $\|T\| \leqq 1$. Then for any $f \in L_{1}$, $p \in L_{1}, p \geqq 0$,

$$
\lim _{n \rightarrow \infty} \frac{\sum_{k=0}^{n} T^{k} f}{\sum_{k=0}^{n} T^{k} p}
$$

exists and is finite a.e. on $\left\{x \mid 0<\sum_{k=0}^{\infty} T^{k} p(x) \leqq \infty\right\}$.

In the present note we show that a result which is stronger than Brunel's lemma can be obtained from Lemmas 1 and 2 of [2], which are the essential two lemmas used in [2] to prove Theorem 1. These two lemmas are also needed to identify the limit (1), as is shown in [3]. It would seem, therefore, that in order to give a complete set of results by the shortest and most direct route one should start with Lemmas 1 and 2 of [2], obtain the existence of the limit (1) by Lemma 3 of the present note and then identify this limit following the method of [3].

We state Lemmas 1 and 2 of [2], in a slightly modified form, as follows.

Lemma 1. Let $T$ be an operator satisfying the hypotheses of Theorem 1. Let $F=g-h, g \geqq 0, h \geqq 0, g \in L_{1}, h \in L_{1}$ and

$$
\sup _{n \geq 0} \sum_{k=0}^{n} T^{k} F>0 \quad \text { a.e. on a set } B \text {. }
$$

Then there exist sequences, $\left\{d_{k}\right\}$ and $\left\{g_{k}\right\}$, of non-negative functions in $L_{1}$, such that

(i) $\sum_{k=0}^{n} \int d_{k}+\int g_{n} \leqq \int g$,

(ii) $\sum_{k=0}^{\infty} d_{k} \leqq h$ a.e. and $\sum_{k=0}^{\infty} d_{k}=h$ a.e. on $B$,

(iii) $T^{n} g=\sum_{k=0}^{n} T^{n-k} d_{k}+g_{n}$.

Received by the editors, February 19, 1964.

1 Corinna Borden Keen Research Fellow at Brown University. 
Lemma 2. Let $T$ be an operator satisfying the hypotheses of Theorem 1. Then for any $g \in L_{1}, h \in L_{1}, h \geqq 0$ and for any integer $j$ we have

$$
\lim _{n \rightarrow \infty} \frac{T^{n+j} g}{\sum_{k=0}^{n} T^{k} h}=0, \quad n \geqq \max (0,-j),
$$

a.e. on $\left\{x \mid 0<\sum_{k=0}^{\infty} T^{k} h(x) \leqq \infty\right\}$.

From these two lemmas we obtain the following:

Lemma 3. Let $T$ satisfy the hypotheses of Theorem 1. For any $f \in L_{1}$, define

$$
E_{f}^{\prime}=\left\{x \mid \limsup _{n \rightarrow \infty} \sum_{k=0}^{n} T^{k} f(x)>0\right\} .
$$

Then, to any measurable set $E$, one can associate a function $\psi_{E} \in L_{\infty}$ such that

$$
E \subset E_{f}^{\prime} \quad \text { implies } \int \psi_{E} f \geqq 0 \text {. }
$$

Brunel's Lemma is obtained from this lemma if the set $E_{f}^{\prime}$ is replaced by the set $E_{f}$ defined as

$$
E_{f}=\left\{x \mid \sup _{n \geqq m} \sum_{k=m}^{n} T^{k} f(x)>0, \text { for all } m \geqq 0\right\} .
$$

It is clear that $E_{f} \subset E_{f}^{\prime}$.

Proof of Lemma 3. Define $\psi_{E}$ as follows [1]:

$$
\psi_{E}(x)=\lim _{n \rightarrow \infty} \psi_{E}^{(n)}(x)
$$

with $\psi_{E}^{(0)}=\chi, \psi_{E}^{(n)}=\chi \bigvee S \psi^{(n-1)}, n \geqq 1$, where $\chi$ is the characteristic function of $E$, and $S: L_{\infty} \rightarrow L_{\infty}$ denotes the adjoint transformation of $T$. Since $S \geqq 0$ and $\|S\|_{\infty} \leqq 1$, we obtain that, for any $f \in L_{1}$,

$$
\int \psi_{E} f=\int \sum_{k=0}^{\infty} \alpha_{k}=\sum_{k=0}^{\infty} \int \alpha_{k},
$$

where the sequence $\left\{\alpha_{k}\right\}$ is defined as

$$
\begin{array}{ll}
\alpha_{0}=\chi f ; & \beta_{0}=\chi^{\prime} f, \\
\alpha_{k}=\chi T \beta_{k-1} ; & \beta_{k}=\chi^{\prime} T \beta_{k-1}, \quad k \geqq 1,
\end{array}
$$


$\chi^{\prime}=1-\chi$ being the characteristic function of the complement of $E$. With usual notations, let $f=f^{+}-f^{-}$and let $\left\{\alpha_{i}^{(+)}\right\},\left\{\beta_{i}^{(+)}\right\},\left\{\alpha_{i}^{(-)}\right\}$, $\left\{\beta_{i}^{(-)}\right\}$be the sequences which are defined as above, but starting from $f^{+}$and $f^{-}$, respectively. To prove the lemma it is sufficient to show that

$$
\sum_{i=0}^{j} \int \alpha_{i}^{(-)} \leqq a \sum_{i=0}^{\infty} \int \alpha_{i}^{(+)},
$$

for any integer $j \geqq 0$, and for any real number $a>1$.

Let $g=a f^{+}$and $h=\sum_{i=0}^{j} \alpha_{i}^{(-)}$, where $a>1$ and $j \geqq 0$ are fixed, and consider $F=g-h$. We will show that

$$
\sup _{n \geqq 0} \sum_{k=0}^{n} T^{k} F>0 \quad \text { a.e. on } E \text {. }
$$

First note that

$$
\chi T^{n} \bar{f}=\chi \sum_{k=0}^{n} T^{n-k} \alpha_{k}^{(-)},
$$

which follows from definition (2). Therefore, using the positiveness of $T$ and $f^{-}$, we have

$$
\chi \sum_{k=0}^{n} T^{k} h=\chi \sum_{k=0}^{n} T^{k} \sum_{i=0}^{j} \alpha_{i}^{(-)} \leqq \chi \sum_{k=0}^{n+j} T^{k} f^{-} .
$$

Now (4) is trivial on the set where $\sum_{k=0}^{\infty} T^{k} h=0$. On the set $E \cap\left\{x \mid 0<\sum_{k=0}^{\infty} T^{k} h\right\}$, by virtue of (5),

$$
\underset{n \rightarrow \infty}{\limsup } \frac{\sum_{k=0}^{n} T^{k} g}{\sum_{k=0}^{n} T^{k} h} \geqq \limsup _{n \rightarrow \infty} \frac{\sum_{k=0}^{n} T^{k} g}{\sum_{k=0}^{n+j} T^{k} f^{-}}=a \limsup \frac{\sum_{n=0}^{n+j} T^{k} f^{+}}{\sum_{k=0}^{n+j} T^{k} f^{-}},
$$

where the last equality follows from Lemma 2. But

$$
\underset{n \rightarrow \infty}{\limsup }\left[\frac{\sum_{k=0}^{n} T^{k} f^{+}}{\sum_{k=0}^{n} T^{k} f^{-}}-1\right]=\limsup _{n \rightarrow \infty} \frac{\sum_{k=0}^{n} T^{k} f}{\sum_{k=0}^{n} T^{k} f^{-}} \geqq 0,
$$

from the hypothesis that $E \subset E_{f}^{\prime}$. Hence, 


$$
\limsup _{n \rightarrow \infty} \frac{\sum_{k=0}^{n} T^{k} g}{\sum_{k=0}^{n} T^{k} h}>1 \text { or } \quad \limsup _{n \rightarrow \infty} \frac{\sum_{k=0}^{n} T^{k} F}{\sum_{k=0}^{n} T^{k} h}>0
$$

which implies (4). Therefore we can apply Lemma 1 to $F$. Since $\chi h=h, \chi^{\prime} h=0$, the $d_{k}$ 's are zero outside $E$ and assertion (ii) of Lemma 1 yields

$$
\sum_{k=0}^{\infty} \int d_{k}=\int h=\sum_{i=0}^{j} \int \alpha_{i}^{(-)}
$$

The proof will be concluded by showing that

$$
\sum_{k=0}^{n} \int d_{k} \leqq a \sum_{k=0}^{n} \int \alpha_{k}^{(+)}, \text {for all } n \geqq 0,
$$

i.e., that (6) implies (3).

To prove (7) first note that

$$
\begin{aligned}
g & =d_{0}+g_{0}, \\
T g_{n} & =d_{n+1}+g_{n+1}, \quad n \geqq 0,
\end{aligned}
$$

which follows from (iii). Similarly definitions of $\alpha_{n}^{(+)}, \beta_{n}^{(+)}$and $g$ imply that

$$
\begin{aligned}
g & =a \alpha_{0}^{(+)}+a \beta_{0}^{(+)}, \\
a T \beta_{n}^{(+)} & =a \alpha_{n+1}^{(+)}+a \beta_{n+1}^{(+)} .
\end{aligned}
$$

Hence

$$
\begin{aligned}
g_{0}-a \beta_{0}^{(+)} & =a \alpha_{0}^{(+)}-d_{0}, \\
g_{n+1}-a \beta_{n+1}^{(+)} & =T\left(g_{n}-a \beta_{n}^{(+)}\right)+\left(a \alpha_{n+1}^{(+)}-d_{n+1}\right),
\end{aligned}
$$

and

$$
\begin{aligned}
\chi^{\prime}\left(g_{0}-a \beta_{0}^{(+)}\right) & =0, \\
\chi^{\prime}\left(g_{n+1}-a \beta_{n+1}^{(+)}\right) & =\chi^{\prime} T\left(g_{n}-a \beta_{n}^{(+)}\right) ;
\end{aligned}
$$

also

$$
\chi\left(g_{n}-a \beta_{n}^{(+)}\right)=\chi g_{n} \geqq 0, \quad n \geqq 0,
$$

which shows that 


$$
g_{n}-a \beta_{n}^{(+)} \geqq 0, \quad n \geqq 0
$$

Now

$$
0=\int\left[\left(a \alpha_{0}^{(+)}-d_{0}\right)-\left(g_{0}-a \beta_{0}^{(+)}\right)\right]
$$

and

$$
\begin{aligned}
\int[ & \left.\sum_{k=0}^{n}\left(a \alpha_{k}^{(+)}-d_{k}\right)-\left(g_{n}-a \beta_{n}^{(+)}\right)\right] \\
& \leqq \int\left[\sum_{k=0}^{n}\left(a \alpha_{k}^{(+)}-d_{k}\right)-T\left(g_{n}-a \beta_{n}^{(+)}\right)\right] \\
& =\int\left[\sum_{k=0}^{n+1}\left(a \alpha_{k}^{(+)}-d_{k}\right)-\left(g_{n+1}-a \beta_{n+1}^{(+)}\right)\right],
\end{aligned}
$$

or, by induction,

$$
0 \leqq \int\left[\sum_{k=0}^{n}\left(a \alpha_{k}^{(+)}-d_{k}\right)-\left(g_{n}-a \beta_{n}^{(+)}\right)\right],
$$

for all $n \geqq 0$, which proves (7).

Acknowledgment. The author is grateful to Professor R. V. Chacon for his comments and for very illuminating discussions on the subject.

\section{BIBLIOGRAPHY}

1. A. Brunel, Sur un lemme ergodique voisin du lemme de $E$. Hopf, et sur une de ses applications, C. R. Acad. Sci. Paris 256 (1963), 5481-5484.

2. R. V. Chacon and D. S. Ornstein, $A$ general ergodic theorem, Illinos J. Math. 4 (1960), 153-160.

3. R. V. Chacon, Identification of the limit of operator averages, J. Math. Mech. 11 (1962), 961-968.

\section{BROWN UNIVERSITY}

